\title{
ESTUDO DA ANISOTROPIA DA SUPERLIGA DE NÍQUEL 718*
}

\author{
Bráulio Pinto Terra ${ }^{1}$ \\ Renata Nunes Oliveira ${ }^{2}$ \\ Matheus Campolina Mendes ${ }^{3}$ \\ Leonardo Sales Araújo ${ }^{4}$
}

\section{Resumo}

Devido à sua vasta aplicação, o estudo das propriedades mecânicas das superligas de níquel é de grande importância. A superliga 718 possui papel fundamental no funcionamento de grades espaçadoras em reatores nucleares. As grades espaçadoras são oriundas de chapas laminadas, submetidas a tratamentos térmicos de solubilização e de envelhecimento. $O$ objetivo do presente trabalho é analisar a anisotropia das propriedades mecânicas de amostras de 0,3mm de espessura, tratadas termicamente por solubilização e envelhecimento, e testadas por tração uniaxial. Além disso, obteve-se os expoentes de encruamento nas condições estudadas, bem como o índice de anisotropia. Foi possível observar maiores valores de limite de escoamento e de alongamento para as amostras transversais provenientes de ambos tratamentos térmicos, enquanto o limite de resistência apresentou comportamento contrário, sendo os maiores valores oriundos das amostras longitudinais.

Palavras-chave: Superliga 718; Anisotropia; Laminação; Tratamentos térmicos.

\section{Abstract}

\section{STUDY OF ANISOTROPY OF 718 NICKEL-BASED SUPERALLOY}

Due to its wide application, the study of the mechanical properties of nickel-based superalloys is of great importance. The 718 superalloy plays a key role as spacer grids in nuclear reactors. The spacer grids are produced from rolled sheets, subjected to thermal treatments of solution annealing and aging. The objective of the present work is to analyze the anisotropy of the mechanical properties of samples of $0.3 \mathrm{~mm}$ thickness, thermally treated by solution annealing and aging, and analyzed by tensile tests. In addition, it was obtained the strain-hardening exponent under the conditions studied, as well as the anisotropy index. It was possible to observe higher values of flow limit and elongation for the transverse samples from both thermal treatments, while the tensile strength showed opposite behavior, being the higher values for the longitudinal samples.

Keywords: 718 Superalloy; Anisotropy; Rolling; Heat treatments.

1 Engenheiro de Materiais, Departamento de Engenharia Química, Universidade Federal Rural do Rio de Janeiro, Seropédica, Rio de Janeiro, Brasil.

2 Engenheira de Materiais, Doutora em Engenharia de Materiais, Professora Adjunta, Departamento de Engenharia Química, Universidade Federal Rural do Rio de Janeiro, Seropédica, Rio de Janeiro, Brasil.

3 Engenheiro de Materiais, Mestre em Ciências dos Materiais, Professor, EBTT, CEMEC, CEFETRJ, Rio de Janeiro, Rio de Janeiro, Brasil.

4 Engenheiro Metalúrgico, Doutor em Engenharia Metalúrgica e de Materiais, Professor Adjunto, COPPE, Universidade Federal do Rio de Janeiro, Rio de Janeiro, Rio de Janeiro, Brasil. 


\section{INTRODUÇÃO}

As superligas de níquel são amplamente utilizadas nas indústrias petroquímica, aeroespacial e nuclear devido às suas propriedades particulares, como a possibilidade de aplicação em uma ampla faixa de temperaturas desde criogênicas até altas temperaturas, resistência à ambientes corrosivos e sobretudo à sua elevada resistência à fadiga [1]. Neste contexto se destaca sua aplicação como material das grades espaçadoras utilizadas nos reatores de nucleares. A grade espaçadora é uma das mais importantes partes do elemento combustível, e é confeccionada a partir de tiras de superliga de níquel 718 entrelaçadas, retiradas transversalmente da direção de laminação da chapa [2].

Devido a este específico uso, há a necessidade de obter e de melhorar as propriedades apresentadas pela superliga 718 por meio de tratamentos térmicos. As fases mais comumente encontradas na superliga 718 são $\gamma, \gamma^{\prime}, \gamma^{\prime \prime}, \delta$ e carbonitretos. $A$ fase $y$ corresponde à matriz austenítica de níquel. As fases $\gamma^{\prime}$ e, especialmente, a fase $\mathrm{Y}^{\prime \prime}$ promovem o endurecimento da superliga por precipitação de partículas de segunda fase. Uma das formas de se controlar o crescimento de grãos é a partir do controle da precipitação da fase $\delta$, que dificulta o escorregamento dos mesmos. Os carbonitretos mais comumente encontrados nesta superliga são os carbonitretos de nióbio $(\mathrm{NbC})$, de titânio $(\mathrm{TiC})$ e o $(\mathrm{Ni}, \mathrm{Ti}) \mathrm{C}[3,4]$.

A laminação é largamente utilizada nestas ligas visto que sua rentabilidade e controle dimensional são bastante elevados. Usualmente, as superligas de níquel são processadas por laminação a quente, numa faixa de temperatura de $900^{\circ} \mathrm{C}$ a $1120^{\circ} \mathrm{C}$. Além disso, o último passo do processamento deve ser de laminação a frio, permitindo melhor controle dimensional e endurecimento por encruamento [3].

De acordo com sua aplicação, a superliga 718 pode ser submetida à tratamentos térmicos de solubilização e/ou a tratamento de envelhecimento, para a obtenção de propriedades e microestruturas desejáveis. O principal objetivo da solubilização consiste na dissolução de partículas de secunda fase. $O$ segundo passo, 0 tratamento de envelhecimento, tem como objetivo a precipitação de $\mathrm{Y}^{\prime} \mathrm{e} \mathrm{Y}^{\prime \prime}$, aumentando a resistência mecânica da liga [5].

A habilidade de controlar a microestrutura da liga faz com que seja possível obter altos valores de limite de escoamento e de tensões de ruptura, podendo-se controlar também sua ductilidade [6].

O endurecimento da liga pode ser causado por diversos fatores, como energia de falha de empilhamento, formação de discordâncias, refinamento de grãos, etc [7]. Para melhor entender as mudanças microestruturais durante o endurecimento da liga, é fundamental saber a quais tensões a liga será submetida quando conformada [8].

Outra consideração importante que deve ser feita no estudo das superligas é seu comportamento durante a deformação plástica uniforme e seu expoente de encruamento [7]. Existem diversos modelos para o cálculo deste, onde tradicionalmente a curva obtida é baseada no modelo de Ludwik $\left(\sigma=K . \epsilon^{n}\right)$. Ludwigson propôs um novo modelo para metais CFC com baixa energia de falha de empilhamento, equação (1), em que há uma mudança na inclinação da curva com o decorrer da deformação [9].

$$
\sigma=K_{1} \cdot \varepsilon^{n_{1}}+e^{K_{2}} \cdot e^{n_{2} \cdot \varepsilon}
$$

$\sigma=$ tensão verdadeira 
$\varepsilon=$ deformação verdadeira

$n_{1}=$ expoente de encruamento em altas deformações

$n_{2=}$ expoente de encruamento em baixas deformações

$K_{1}=$ constante de proporcionalidade em altas deformações

$K_{2}=$ constante de proporcionalidade em altas deformações

Metais geralmente apresentam comportamento anisotrópico quando testados em tração uniaxial [10] e este comportamento pode ser atribuído à textura do material, que por sua vez pode ser causada pelo processamento termo-mecânico de chapas finas [11]. Mehta et al. [12] estudaram a liga Ni-20Cr-8Fe e relataram a presença de anisotropia devido a textura cristalográfica. Para quantificar a anisotropia, Wu et al. [13] propuseram um modelo matemático, usando o alongamento calculado no teste de tração uniaxial, conforme equação (2),

$$
\delta=100 \frac{|\% \mathrm{El}(\mathrm{L})-\% \mathrm{El}(\mathrm{T})|}{\% \mathrm{El}(\mathrm{L})+\% \mathrm{El}(\mathrm{T})}
$$

onde \%El(L) e \%El(T) são os alongamentos nas direções longitudinais e transversais, respectivamente, e $\delta$ é o índice de anisotropia.

O objetivo do presente estudo é analisar a influência do processo de laminação na anisotropia de propriedades mecânicas da superliga 718 submetida aos tratamentos térmicos de solubilização e envelhecimento.

\section{MATERIAIS E MÉTODOS}

\subsection{Preparação das amostras}

Amostras da superliga 718 foram retiradas de uma chapa fina, previamente laminada a frio (espessura de $0,3 \mathrm{~mm}$ e comprimento de $25 \mathrm{~mm}$ ), apresentando composição química conforme Tabela 1. Em adição, as mesmas foram retiradas tanto no sentido longitudinal de laminação, $0^{\circ}(\mathrm{DL})$, bem como no sentido transversal de laminação, $90^{\circ}$ (DT), sendo submetidas à tratamentos térmicos de solubilização $\left(975^{\circ} \mathrm{C}\right.$ por $1 \mathrm{~h}$ e $10 \mathrm{~min}$, seguido de resfriamento em água, e envelhecimento $\left(760^{\circ} \mathrm{C}\right.$ por $4 \mathrm{~h}$ e $45 \mathrm{~min}$, resfriamento no forno com taxa de de $37^{\circ} \mathrm{C} / \mathrm{h}$, seguido de resfriamento em água). As amostras foram nomeadas DLS e DTS, para as solubilizadas nas direções longitudinal e transversal, respectivamente, e DLE e DTE para as envelhecidas nas direções longitudinais e transversais, respectivamente.

Tabela 1: Composição química da superliga 718 (\% em massa, exceto o N, que está representado

\begin{tabular}{|l|l|l|l|l|l|l|}
\hline $\mathrm{Ni}$ & $\mathrm{Cr}$ & $\mathrm{Fe}$ & $\mathrm{Ti}$ & $\mathrm{Al}$ & Mn & $\mathrm{Si}$ \\
\hline $50-55$ & 21 & Balanço & $0,65-1,15$ & $0,20-0,80$ & Máx. 0,35 & Máx. 0,35 \\
\hline $\mathrm{P}$ & $\mathrm{S}$ & $\mathrm{Mo}$ & $\mathrm{Cu}$ & $\mathrm{C}$ & Co & $\mathrm{N}(\mathrm{ppm})$ \\
\hline 0,015 & 0,015 & $2,8-3,3$ & Máx. 0,30 & Máx. 0,08 & Máx. 1,00 & $70-150$ \\
\hline
\end{tabular}

\subsection{Microscopia Eletrônica de Varredura}

Para analisar a distribuição dos carbonitretos nas amostras, foram obtidas imagens através do equipamento MEV JSM 6460LV de marca JEOL disponível no Laboratório Multiusuário de Microscopia Eletrônica e Microanálise do COPPE/UFRJ. 
Foram realizadas fractografias para avaliar o mecanismo e morfologia de fratura, além da identificação da distribuição dos carbonitretos nas superfícies, fazendo-se uso do equipamento MEV VEGA 3LMU de marca TESCAN do Laboratório Multiusuário de Microscopia Eletrônica e Microanálise do COPPE/UFRJ. As amostras foram anteriormente levadas ao aparelho ultrassom AROTEC para limpeza da superfície.

A textura da liga e tamanho de grãos foi avaliada por difração de elétrons retroespalhados (EBSD), utilizando-se o microscópio eletrônico de varredura Cambridge CAM-SCAN, disponível no Laboratório de Caracterização de Materiais do CEFET-RJ. Utilizou-se o software MTEX no Matlab para os cálculos. Além disso, foram calculados os números de interceptos entre uma grade criada sobre as imagens e os contornos de grãos de tamanhos superiores a $19 \mu \mathrm{m}$. A análise foi feita utilizando o software ImageJ.

\subsection{Ensaio mecânico}

Três corpos de prova de cada condição (DLS, DTS, DLE, DTE) foram submetidos à ensaios de tração uniaxial $O$ ensaio foi realizado utilizando o aparelho EMIC DL1000, célula de carga de $100 \mathrm{KN}$, e taxa de deformação de 6,5 x 10-4 s-1. O limite de escoamento (LE), o limite de resistência (LR) e o alongamento $(\varepsilon)$ foram obtidos. Os resultados foram avaliados na região de deformação plástica da curva tensãodeformação verdadeiras, pela relação proposta por Ludwigson (Equação 1), a fim de se obter o expoente de encruamento durante a deformação.

Todos os dados foram avaliados foram submetidos à Análise de Variância (ANOVA) com um fator (tratamento térmico) e dois níveis de fator (direção de laminação), utilizando o teste Tukey para comparação de médias, foram utilizados os níveis de confiança de $95 \%$, 90\% e $80 \%$. Para tal, utilizou-se o software OriginPro8.

Utilizou-se a equação (2), para cálculo da anisotropia apresentada pelas amostras submetidas ao ensaio de tração, de acordo com o sentido de laminação da chapa.

\section{RESULTADOS E DISCUSSÃO}

\subsection{Microestrutura}

De acordo com a figura 1, foi possível observar que os grãos sofreram refinamento nas regiões de precipitação de carbonitretos. A imagem é obtida a partir da separação de grãos iguais ou menores que 19 microns e maiores que 19 microns. É possível observar também que os grãos sofreram leve alinhamento de acordo com a direção de processamento da chapa, conforme figura 2. Verificou-se que existem 179 interceptos na direção longitudinal e 243 interceptos na direção transversal. Tais fatos podem estrar contribuindo para o maior limite de escoamento na direção transversal, visto que o maior número de barreiras (contornos) para tal direção, dificulta o movimento de discordâncias. A figura 3 apresenta a textura cristalográfica do material. 

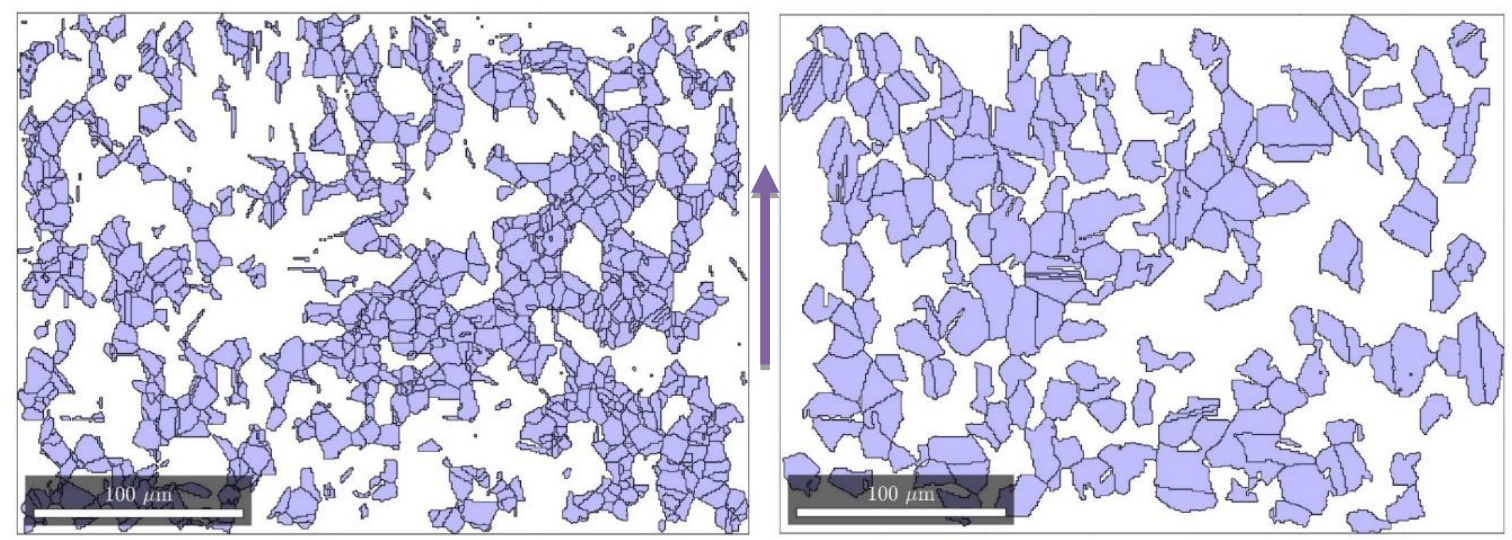

Figura 1. À esquerda, identificação de grãos com tamanhos menor ou igual a 19 micrometros e à direita, identificação de grãos com tamanhos superior a 19 micrometros.
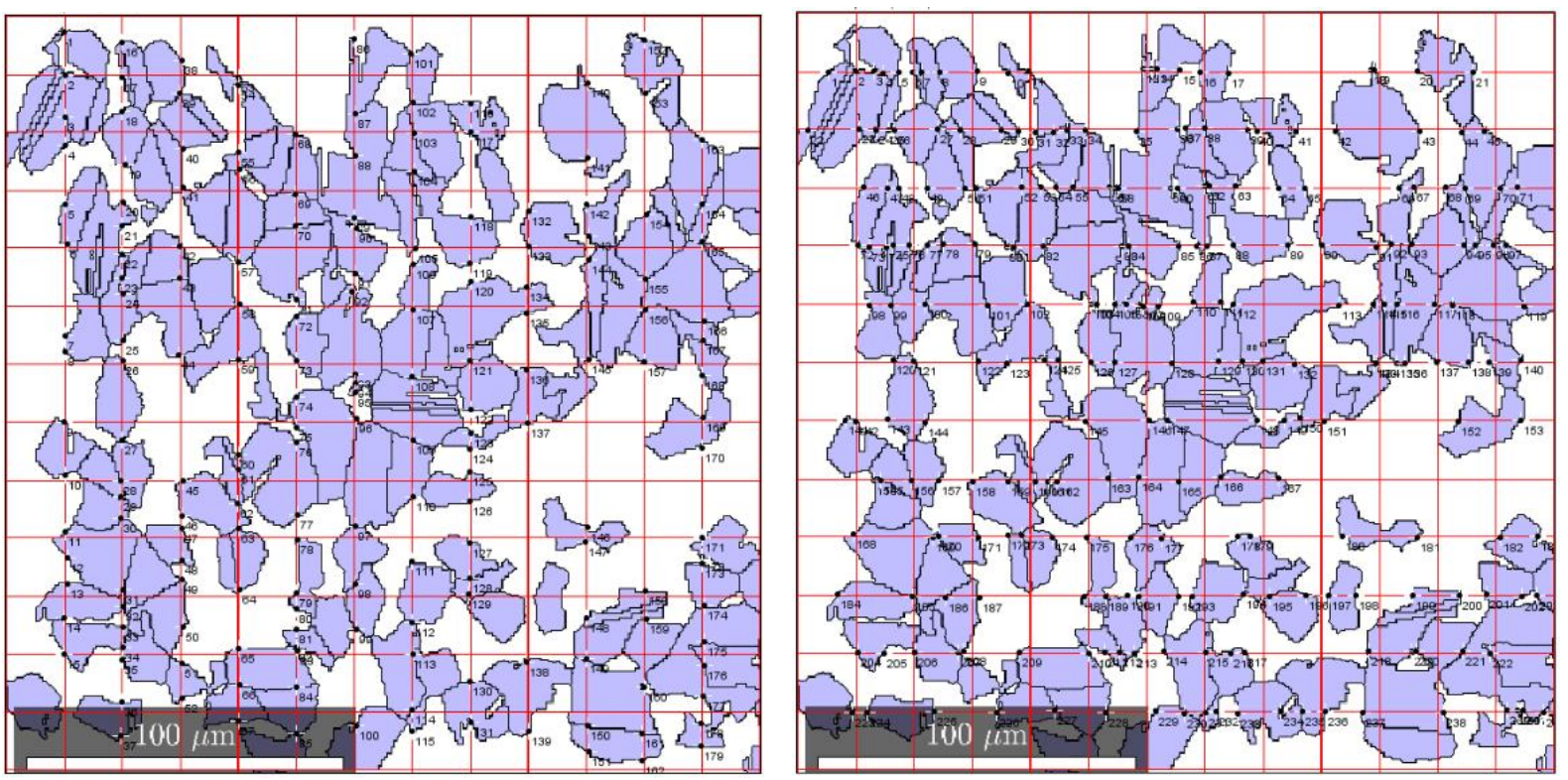

Figura 2. Método de interceptos para análise qualitativa de alongamento do grão nas direções longitudinal (à esquerda) e transversal (à direita).
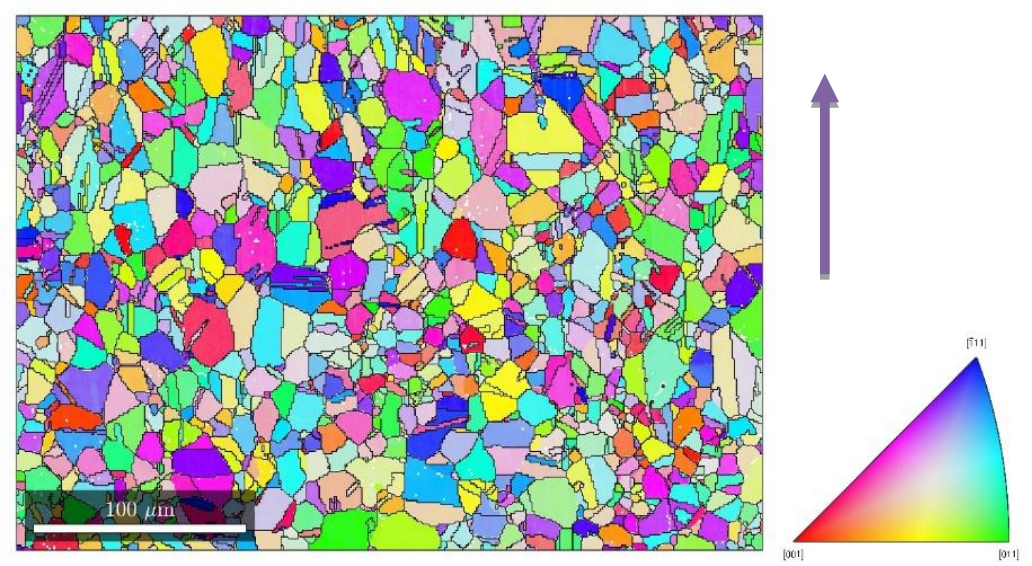

Figura 3. Figura de polo invertida da amostra da amostra de superliga 718 estudada. Direção de laminação é indicada ao lado da imagem pela seta amarela.

\subsection{Ensaio Mecânico}


Os resultados dos ensaios mecânicos são apresentados na tabela 2 e 3.

Tabela 2. Propriedades mecânicas das amostras tratadas termicamente de diferentes direções.

\begin{tabular}{|c|c|c|c|}
\hline Amostra & $\begin{array}{c}\text { Limite de escoamento } \\
(\mathrm{MPa})\end{array}$ & Alongamento & $\begin{array}{c}\text { Limite de resitência } \\
(\mathrm{MPa})\end{array}$ \\
\hline DLS & $399,0 \pm 13,4$ & $0,45 \pm 0,03$ & $824,2 \pm 4,4$ \\
\hline $\mathrm{DTS}$ & $419,8 \pm 1,3$ & $0,51 \pm 0,01$ & $817,9 \pm 1,0$ \\
\hline DLE & $1046,9 \pm 30,5$ & $0,14 \pm 0,01$ & $1345,5 \pm 8,3$ \\
\hline DTE & $1104,6 \pm 8,3$ & $0,15 \pm 0,01$ & $1315,5 \pm 3,3$ \\
\hline
\end{tabular}

É possível notar maiores valores de limite de escoamento (LE) nas amostras transversais tanto para a condição solubilizada quanto para a envelhecida, mais especificamente $5,22 \%$ e $5,51 \%$ maiores que os valores das amostras longitudinais DLS e DLE, respectivamente. Em outros estudos com superligas de níquel, resultados diferentes de LE foram encontrados, onde os valores na direção longitudinal foram maiores que aqueles na direção transversal [12]. Estudos [12] atribuem esta diferença à composição da liga, bem como às características microestruturais, como tamanho de grão e sua distribuição, morfologia e também à textura cristalográfica.

Por outro lado, os limites de resistência (LR) apresentaram maiores valores nas amostras longitudinais. Neste caso, a diferença foi de $0,72 \%$ para as amostras solubilizadas (UTSDLS > UTSDTS), e de $2,23 \%$ para as amostras envelhecidas (UTS DLE > UTS DTE), respectivamente. O mesmo comportamento foi encontrado em superliga de níquel previamente estudada [12,14,15].

Com relação ao alongamento, as amostras transversais apresentaram maiores valores frente as amostras longitudinais, de $11,69 \%$ e $8,87 \%$ para amostras solubilizadas $\left(\varepsilon D L S<\varepsilon D T S\right.$ ) e envelhecidas ( $\varepsilon_{\text {DLE }}<\varepsilon$ DTE), respectivamente. Apesar dos limites de escoamento apresentarem valores distintos entre as diferentes direções estudadas, eles mostraram-se ser diferentes estatisticamente somente no nível de confiança de $90 \%(f=0,1)$ para as amostras solubilizadas e de $95 \%(f=0,005)$ para as envelhecidas, através do teste Tukey. Já para o limite de resistência, o teste indicou diferença estatística entre os resultados em nível de confiança de $90 \%(\mathrm{f}=0,1)$ para amostras solubilizadas e de $95 \%(f=0,005)$ para as envelhecidas. Para os valores de alongamento, houve diferença estatística no nível de confiança de $90 \%(\mathfrak{f}=0,1)$ para amostras solubilizadas, enquanto não foi identificado diferença estatística em nenhum dos três níveis de confiança estudados para as envelhecidas.

Variações de propriedades mecânicas com direção de processamento em superligas foram analisados em outros trabalhos [12, 15]. Para Mehta et al. [12], a liga Ni-20Cr$8 \mathrm{Fe}$ foram observados maiores valores de escoamento e resistência nas amostras longitudinais, enquanto a ductilidade foi maior nas amostras transversais. $\mathrm{O}$ mesmo comportamento observado para a liga $\mathrm{Ni}-16 \mathrm{Cr}$ [15]. Para a liga $\mathrm{Ni}-16 \mathrm{Mo}$ foram observados maiores valores de limite de escoamento na direção longitudinal, enquanto o limite de resistência e o alongamento foram maiores na direção transversal [15]. Tais diferenças foram atribuídas à variações microestruturais, como tamanho e morfologia de grãos e segundas fases, bem como à textura cristalográfica observada. No caso da presente liga, os valores de escoamento foram maiores para as amostras longitudinais. Tal diferença pode estar relacionada as formações de carbonitretos alinhados na direção de processamento (laminação). 
Ao redor de tais regiões, há uma tendência a um refino de grão localizado, o que pode influenciar o início do escoamento do material. Tal aspecto pode também influenciar o comportamento de encruamento bem como a nucleação de microcavidades no processo de fratura. Nesse sentido, os valores de encruamento das duas amostras foram determinados. Para isso, a relação de Ludwigson, Equação (1), foi utilizada para calcular os parâmetros envolvidos com o mecanismo de encruamento. Essa relação promove um melhor ajuste para materiais propensos à formação de maclas $[8,14,16]$. Os resultados são apresentados na tabela 3 .

Tabela 3. Valores de expoentes de encruamento e constantes.

\begin{tabular}{|c|c|c|c|c|}
\hline Amostra & $\mathbf{K 1}$ & $\mathbf{n}_{\mathbf{1}}$ & $\mathbf{K 2}$ & $\mathbf{n}_{\mathbf{2}}$ \\
\hline DLS & $1130,6 \pm 58,4$ & $0,71 \pm 0,02$ & $6,0 \pm 0,01$ & $1,31 \pm 0,08$ \\
\hline DTS & $1249,5 \pm 290,7$ & $0,76 \pm 0,08$ & $6,0 \pm 0,01$ & $0,95 \pm 0,44$ \\
\hline DLE & $966,6 \pm 145,8$ & $0,23 \pm 0,10$ & $6,6 \pm 0,36$ & $1,31 \pm 0,73$ \\
\hline DTE & $767,6 \pm 68,6$ & $0,35 \pm 0,06$ & $6,9 \pm 0,03$ & $0,79 \pm 0,14$ \\
\hline
\end{tabular}

Foi observado que as amostras transversais apresentaram maiores valores médios de expoente de encruamento frente às amostras longitudinais, tanto em baixas $\left(n_{2}\right)$ quanto em altas ( $\left.n_{1}\right)$ deformações, assim como calculado por Mehta et al. [12].

Os expoentes de encruamento das amostras solubilizadas não apresentaram diferença estatística relevante para os níveis de confiança estudados, enquanto as amostras envelhecidas apresentaram diferença apenas no nível de confiança de $80 \%(\mathrm{f}=0,2)$.

O cálculo do índice de anisotropia, determinado à partir da equação (2) foi de 5,52 e 4,24 para as amostras solubilizadas e envelhecidas, respectivamente. Tal anisotropia é relacionada aos grãos formados ao longo da direção de processamento, bem como à textura cristalográfica.

\subsection{Análise fractográfica}

Verificou-se que as amostras, em todas condições estudadas, apresentaram fratura do tipo dúctil, evidenciadas pelas microcavidades na superfície da amostra. Pôde-se notar também o alinhamento dos carbonitretos na matriz, conforme previamente discutido.

É possível observar nas amostras DLS microcavidades concentradas de forma pontual, em torno de regiões de distribuição dos carbonitretos. Já nas amostras DTS, foram observadas microcavidades maiores e linear devido a presença dos carbonitretos, conforme Figura 3. Os mesmos comportamentos morfológicos foram observados para as amostras DLE e DTE, conforme apresentado na Figura 4. 

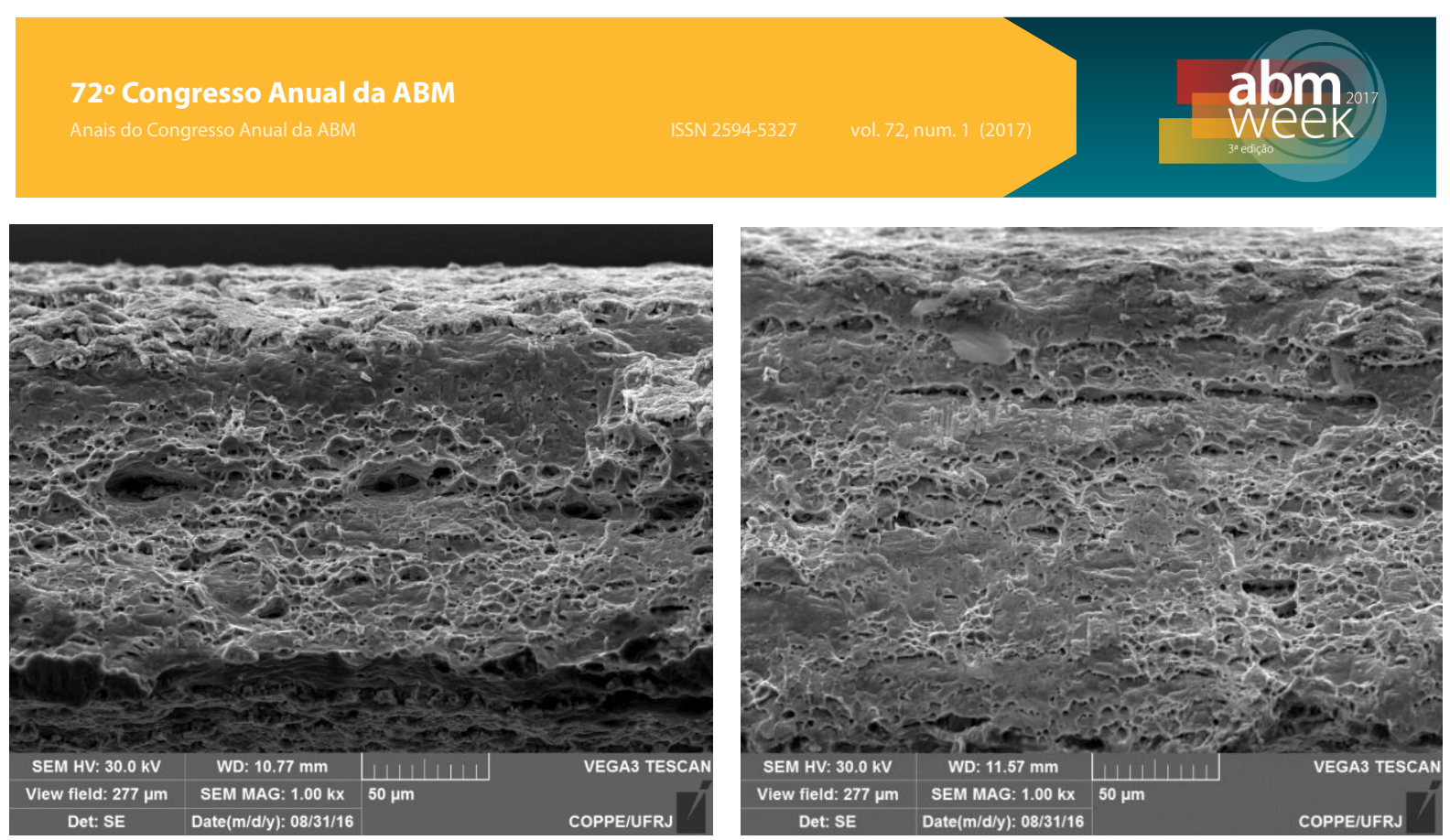

Figura 31. Fractografia de amostra DLS à esquerda e DTS à direita.
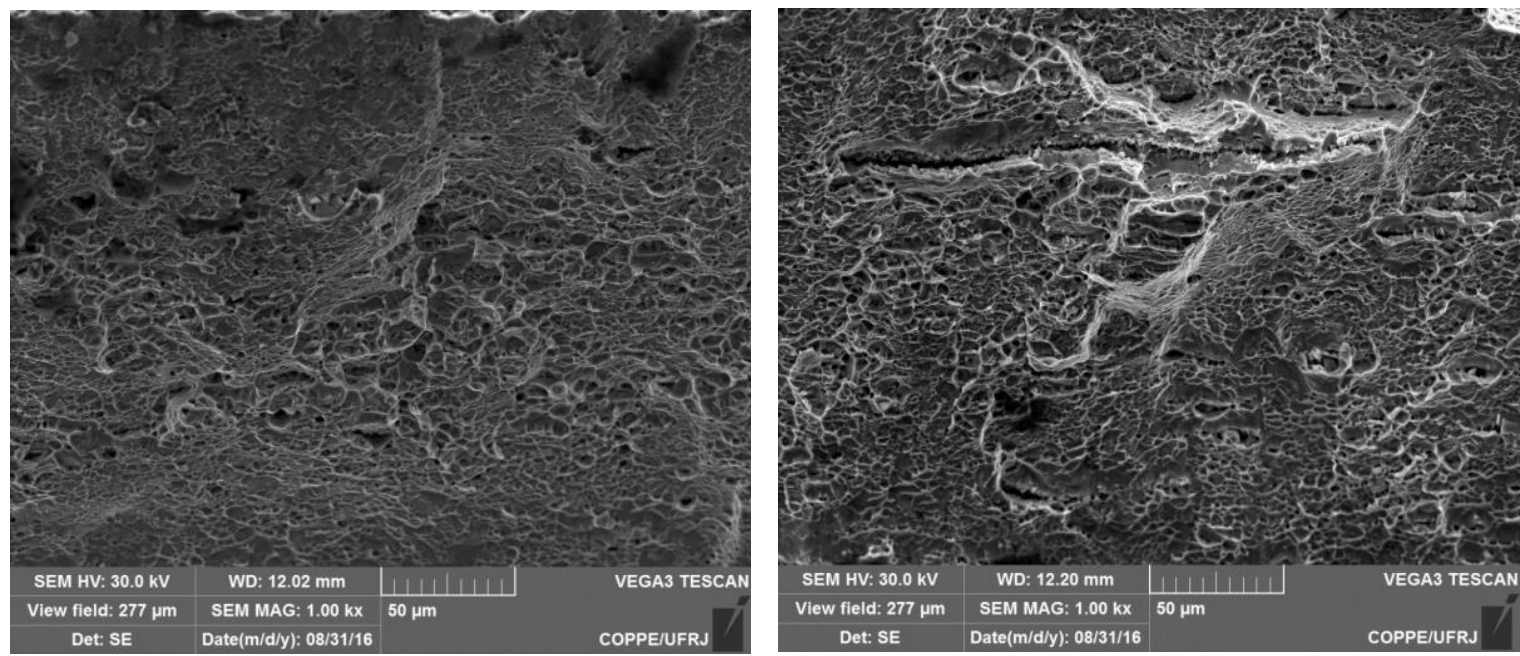

Figura 4. Fractografia de amostra DLE à esquerda e DTE à direita.

\section{CONCLUSÃO}

A superliga 718 apresentou, tanto para a condição solubilizada quanto para a envelhecida, anisotropia de propriedades mecânicas.

O limite de escoamento mostrou-se maior para as amostras longitudinais, ao passo que o limite de resistência, ductilidade e expoentes de encruamentos apresentaram maiores valores para as amostras transversais. Essas diferenças de resistência são atribuídas à mudanças nos tamanhos de grão ao longo da direção de processamento, influenciado pelos agrupamentos de carbonitretos na direção de processamento da chapa. Essas fases também agem como sítios nucleadores de microcavidades, afetando de maneira positiva a ductilidade nas amostras transversais.

A textura cristalográfica observada também pode ter influenciado as variações das propriedades do material. 


\section{Agradecimentos}

Laboratório de Propriedades Mecânicas, COPPE/UFRJ.

Laboratório de Microscopia Eletrônica e Microanálise, COPPE/UFRJ.

Laboratório de Caracterização de materiais, CEFET/RJ.

Pibic/CNPq.

\section{REFERÊNCIAS}

1 REED, R. C., The Superalloys: Fundamentals and Applications, Cambridge: Cambridge University Press, 2006.

2 PERROTTA, J.A., Introdução à Engenharia do Núcleo de Reatores, São Paulo: IPENCNEN/SP, 1999.

3 ARAUJO, L.S., Processamento e Caracterização de Superliga de Níquel 718 Para Aplicações Nucleares, Tese de /doutorado, COPPE/UFRJ, 2012;

4 REZENDE, M.C., Estudo Sobre o Efeito de Diferentes Tratamentos Térmicos na Ocorrência de Envelhecimento Dinâmico no Inconel 718, Tese de Doutorado, COPPE/UFRJ, 2015.

5 MURATA, Y., MORINAGA, M., YUKAWA, N., OGAWA, H., KATO, M., Solidification Structures of Inconel 718 with Microalloying Elements, Superalloys 718, 625, 706 and Various Derivatives, The Minerals, Metals \& Materials Society. Ano [acesso em 2017].

Disponível

em: http://www.tms.org/superalloys/10.7449/1994/Superalloys_1994_81_88.pdf.

AKCA, E, A Review on Superalloys and IN718 Nickel-Based Inconel Superalloy, Periodicals of Engineering and Natural Sciences, 2013; 1:15-27.

7 CORREA, E. C. S., Aspectos do Encruamento de Metais Previamente Deformados a Frio, Tese de doutorado, UFMG, 2004.

8 SATYANARAYANA, D.V.V., SATYA PRASSAD, K., MALAKONDAIAH, G., SARMA, D.S., Strain Hardening Behaviour of an $\mathrm{Fe}-\mathrm{Ni}-\mathrm{Cr}-\mathrm{Al}$ alloy, Materials Science and Technology, 2013; 23(1): 79-86.

9 LUDWIGSON, D.C., Modified Stress-Strain Relation for FCC Metals and Alloys, Metalurgical Transactions, 1971; 2: 1971-2825.

10 DIETER.G.E., Mechanical Metallurgy, SI Metric Edition, London: MacGraw-Hill Book Company, 1988.

11 MARTINS, F. J. S., Efeito dos Tratamentos Térmicos de Solubilização e Envelhecimento na Microestrutura da Liga Inconel 718, Projeto de conclusão de curso, Universidade Federal do Rio de Janeiro, Brasil, 2008.

12 MEHTA, K.K., MUKHOPADHYAY, P., MANDAL, R.K., SINGH, A.K., Mechanical Properties Anisotropy of Cold Rolled and Solution Annealed Ni-20Cr-8Fe Alloy, Materials Science \& Engineering A, 2014; 613: 71-81.

$13 \mathrm{WU}, \mathrm{Y} . \mathrm{T}$., KOO, C. H., Effect of Temperature on the Anisotropic Supperplasticity of Textured Ti-25Al-10Nb Alloy, Scripta Materialia, 1998; 38: 267-271.

14 ANKAMMA, K., SATYANARAYANA, D. V. V., REDDY G. C., KOMARAIAH, M., PRASAD, N. E., In-plane Anisotropy in Tensile Deformation and its Influence on the Drawability of Nimonic c-263 Alloy Sheets, Sadhana, 2011; 36(2): 223-249.

15 MEHTA, K.K., MUKHOPADHYAY, P., MANDAL, R.K., SINGH, A.K., Microstructure, Texture, and Orientation-Dependent Flow Behavior of Binary Ni-16Cr and Ni-16Mo Solid Solution Alloys, The Minerals, Metals \& Materials Society, 2015; 46(A): 36563669. 
16 PRAVEEN, K.V.U., SASTRY, G.V.S., SINGH V., Work-Hardening Behavior of the NiFe Based Superalloy IN718, The Minerals, Metals \& Materials Society, 2007; 39(A): 65-78 